\title{
3-D Ray Tracing Based GPU Accelerated Field Prediction Radio Channel Simulator
}

\author{
Juha Pyhtilä, Pekka Sangi*, Heikki Karvonen, \\ Markus Berg, Rameez Lighari, Erkki Salonen, Aarno Pärssinen, Markku Juntti \\ Centre for Wireless Communications, ${ }^{*}$ Center for Machine Vision and Signal Analysis \\ Faculty of Information Technology and Electrical Engineering, University of Oulu \\ Email: firstname.lastname@oulu.fi
}

\begin{abstract}
A ray tracing simulator for urban and indoor environments is introduced. The simulator uses NVIDIA graphics processing unit (GPU) accelerated CUDA parallel computing platform and programming mode and the OptiX Ray Tracing Engine. As a use case, channel characteristics for Global Navigation Satellite System (GNSS) satellites are simulated and compared with measurements in an urban area. The speedup achieved by parallel processing allows computation of multiple relevant reflections characteristic of a satellite channel.
\end{abstract}

\section{INTRODUCTION}

With the increasingly pervasive wireless connectivity, high rate radio systems adopting multiple-input multipleoutput (MIMO), (pencil) beamforming for mmWave connections, and other advanced transmission techniques, the multi-dispersive characteristics of the radio channel play a crucial role in the network planning and system design. Therefore, three dimensional (3-D) channel knowledge and models are required.

Channel modeling methods can be broadly categorized as statistical and deterministic. Often those approaches are combined in hybrid methods [1]. In pure statistical modeling, different measurement or theory based random distributions (e.g. Rayleigh, Rice, Nakagami) are used to represent the channel behavior coupled with different parameters tuned to the environment specifics [2], [3]. These models have the drawback of being very generic in nature, because the models are an average of a congregate of different environments and that will also cause inaccuracy to the results for different environments. Deterministic methods can be divided into empirical models and site-specific methods. A classic example of empirical modeling is the Okumura-Hata propagation over rooftops for general mobile communications [4], which is derived on the basis of field measurements. These models are accurate only in the environments which are similar to the measurement environment. In contrast, if sufficiently accurate maps of the geometry and materials of the environment are available (a site-specific model), it is possible to apply more generic computational methods, which give solutions for field vectors and other variables. The most exact way to solve a propagation problem would naturally consider the Maxwell equations for the situation via, for example, the finite-difference time-domain (FTDT) or the moment methods. However, such an approach suffers from a huge complexity and the resulting computation time is excessive for most practical applications. An alternative is provided by methods based on asymptotic geometric optics (GO), where the modeling is based on tracing of rays. Thanks to their intrinsic capability to simulate multipath propagation, ray tracing (RT) models seem a good solution to provide an accurate, site-specific field prediction and a multidimensional characterization of radio propagation channel in the time, space, and polarization domains.

To enable fast 3D channel knowledge acquisition, we consider graphical processing unit (GPU) based execution of ray tracing in this work. Especially, NVIDIA's OptiX Ray Tracing Engine [5] provides a convenient platform for implementation. Taking a GPU based approach is partially supported by a study, where GPU implementation was noted to be 2-15 times faster than a corresponding CPU based implementation [6]. GPU's parallel calculation power has even potential for nearly real-time computation [7], which can make the approach applicable not only for network planning but for operation optimization. The simulator is generic in nature, because only electromagnetic theory is needed for its theoretical basis. Although GPU based ray tracing is fairly commonly used for wireless channels [8][9], modelling satellite channels using this approach has not been published according to our best knowledge.

Compared to point to point channels at small distances a satellite transceiver is situated at an extreme distance. For example, the GPS satellites considered in the experiments of this work are located in the range 20000-25000 $\mathrm{km}$ from the receiver. The consequence is that that even for a large receiving area such as a city, the incoming signal may be modelled as a plane wave without notable errors. Considering simulations this is an advantageous viewpoint, because it circumvents complications such as inexactly known positions of satellites, atmospheric and tropospheric effects, and inexact transmitter gain information in the direction the receiver. For individual 
satellites the simulations reduce to the consideration of relative quantities with respect to the line of sight (LOS) signal. If absolute values such as what the signal powers are in reality, then the LOS signal would have to be scaled by looking up at the exact position and gain of the satellite, and the free space path loss equations would have to be used. In this way, different satellites may be compared. Different environmental factors such as weather conditions or troposphere induced delays would naturally affect the reliability of the analysis and would have to be modelled [10].

The simulator will enable an efficient planning of different types of communication networks, since their signal propagation can be accurately modeled based on the ray tracing information. We will introduce a relevant RT background and the proposed solutions for efficient calculation of ray paths. As an example scenario, novel results will be introduced for the ray tracing of global navigation satellite system (GNSS) signals.

\section{Background on Determination of Ray Paths}

Geometric optics approximation means that electromagnetic (EM) radiation propagating in a certain direction may be viewed as a ray tube, which does not interact with the neighboring rays [11], [12]. This is consistent also with the locality of reflections and diffractions. The wavefronts of the ray tube, the electric and magnetic field, are orthogonal to the propagation direction [13]. Using interaction point locations, material geometries and other material properties, an exact output electric field vector at the terminating point of the ray can be calculated. This is done by evaluating a $2 \times 2$ polarimetric transformation matrix [14] using the Fresnel equations and the uniform theory of diffraction (UTD) [15], [16], [17]. Possible scattering can be modeled by a polarimetric transformation matrix with random phase contributions and some pathloss model such as the Lambert model [18]. The transmitted field vector along the ray is multiplied by the polarimetric matrix to obtain the output field vector. The received power can be calculated if the effective antenna length is known. It can either be theoretically obtained or measured.

EM waves are modeled as rays orthogonal to the wave fronts, which have paths drawn in a user defined map of an environment. This map is usually 3-dimensional (3-D), although for speedup purposes the environment can be represented as a so-called $2.5-\mathrm{D}$, model where buildings are modeled by flat roofed objects with vertical walls (cylinders).

Two alternative ray tracing paradigms, which are deterministic ray tracing and shoot and bounce ray tracing (SBR) [3], [13], have been formulated. The conventionally used RT method is the deterministic one, where ray paths from a transmitter to a receiver are computed using analytical geometry. Exponential complexity increase is customary for those. In order to overcome the computational complexity, the SBR method has been developed [19]. Therein rays are shot out from the transmitter in different and usually evenly distributed directions and at each interaction at a structure the outgoing ray(s) are calculated by GO, i.e., a ray is shot and it bounces around the map. For simple planar surfaces, the law of reflection is used to obtain the reflection angle and the Snell's law is used for calculation of the refractive angle in the case that penetration inside walls is to be modeled. The complexity of SBR is linear with respect to the number of elements in the map database of the environment [20]. When modeling communication channels in an urban environment, where many reflections need to taken into account, this linear increase of complexity is a greatly advantageous. Preprocessing of the environment map by various visibility algorithms is not necessary to achieve linear complexity for reflections. With diffractions the situation is different. According to theory, once a ray hits a diffraction edge, it creates a continuum of rays shooting out in the so called Keller cone [15]. Each outgoing ray forms the same angle with the diffracting edge as the incoming ray. It follows again that the complexity grows again exponentially with the number of diffractions allowed on a path. Fortunately, it is usually enough to model only one diffraction, because already a second diffraction occurring for a communication signal diminishes the power significantly. Another consideration concerning diffraction is that there are only a finite number of rays shot from a transmitter. Therefore some sort of capturing surface for diffracting edges has to be modeled. Here we enclose the convex edges of building in cylinders which is the approach used for example in [9]. A ray hitting such a cylinder is considered to be diffracted.

\section{Implementation of Path Computation}

In this work, we adopted the SBR method for GPU based path computation. Especially, we utilize the CUDA based OptiX engine [5], which provides a general purpose ray tracing platform. This approach has been used earlier in the work by Schiller et al.[9], which provided some guidelines for our implementation. In the solution, computation of paths was separated from the EM calculations, which were executed as a postprocessing step in Matlab.

\section{A. Basic Principle}

Implementation of the path computation under the OptiX framework is illustrated in Fig. 1. Rays are launched using the ray generation programs, which are implemented for different transmitter types and for simulating the diffraction as explained in Sec III-D. The rays propagate through the geometry via reflections and information about these interactions is collected to the payload. So-called virtual receiver (VRX) objects, 
associated with the actual receiver points and diffraction edges, are established as spheres and cylinders, respectively. Any hit of a propagating ray with a VRX object generates a path segment, which is buffered and transferred to the host memory.

Finally, the host program removes any duplicates from the set of path segments. Many rays with the same path signature may hit particular receiver sphere, and we keep only that path segment, whose propagation line is closest to the receiver point.

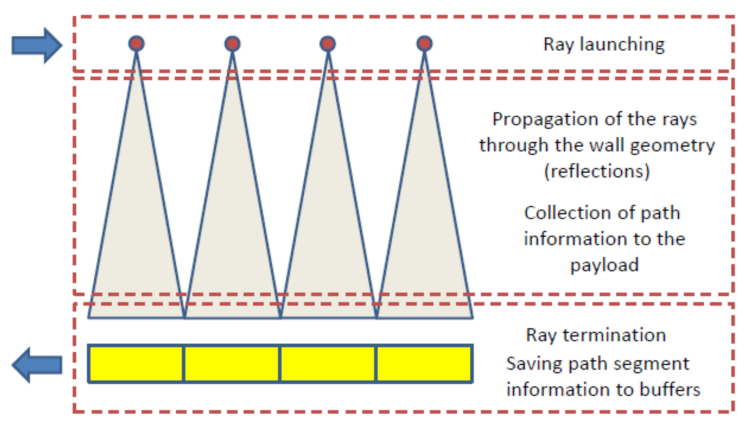

Fig. 1. Ray tracing principle.

\section{B. Path Segments}

Four path segment types for representing paths composed of reflections and diffractions are shown in Fig. 2a. If the path contains just reflections, then it can be represented with one path segment. Diffraction edge cylinder hit points are mapped to launch points, which can subsequently work as starting points for other path segments. The representation of propagation paths containing one diffraction is outlined in Fig. 2 b.

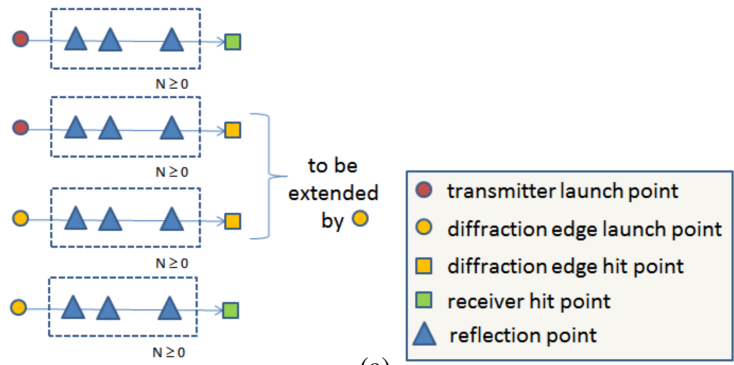

(a)

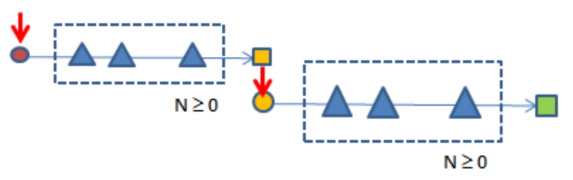

(b)

Fig. 2. Path segments. (a) Four path segment types for dealing with reflection and diffraction. (b) An example of a complete propagation path.

\section{Focusing}

Most of the rays launched do not provide any paths that reach the receiver. As a method for reducing the number of rays launched, Schiller et al. [9] proposed the SBR approach, where the sizes of the receiver spheres and edge cylinders are gradually decreased while the ray sampling density is increased.

In our implementation, we can set the number of iterations $(N)$. In the iterations from 1 to $N-1$, ray directions are flagged active, if there is any path that hits the receiver sphere. In the $N$ th iteration, the sizes of the VRX spheres/cylinders are set to their final values, and the number of path segments for each ray are calculated. This information is then used to reserve buffer slots for path segments, and the slots are filled in the final ray launch.

\section{Ray Launchers}

1) Far Transmitter: In the case of the GNSS simulation, considered in our example use case below, the wavefront reaching the receiver zone is approximately planar, and this idea is adapted to model the radio source. In our implementation, a rectangular planar area is set to cover the geometric area, which is assumed to provide relevant contributions to the communications signal arriving at the receiver. A rectangular lattice of sample points placed over the plane provides origins for the rays, and it is straightforward to apply OptiX 2-D launching with focusing in ray generation. An example is shown in Fig. 3a.
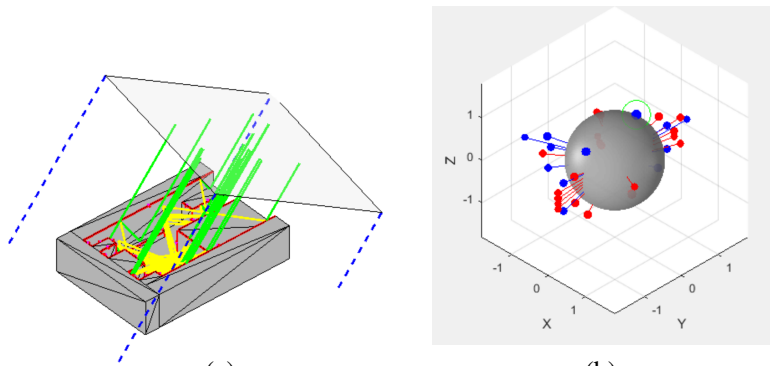

(a)

(b)

Fig. 3. An example of path computation. (a) Launching from a planar wavefront. The rectangle which contains the origins of the rays is placed at some distance over the building geometry. Green and yellow lines show the ray paths that reach the receiver (yellow lines indicate the diffracted rays). (b) Rays arriving to the receiver. The lengths and colors of the needles indicate, respectively, the propagation delay and polarization.

2) Near Transmitter: Transmitters close to the receivers are modelled as point sources. The problem in this case is to sample a sphere in some manner and for this purpose we consider two kinds of approaches. Firstly, it is possible to use a cube-based approach, where each side of a cube is sampled using a rectangular lattice, and the sample points are mapped onto sphere by normalizing the vector lengths. As shown in Fig. 4a, 
this approach leads to slightly non-uniform sampling of the sphere. However, cube-based sampling allows implementation of focusing, which can be an advantage when only some ray directions are relevant.

However, if focusing does not provide benefits, it is straightforward to use spiral sampling, which provides close to uniform sampling of directions. An example is shown in Fig. 4b.

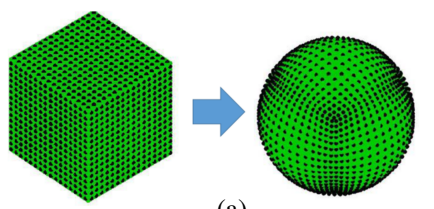

(a)

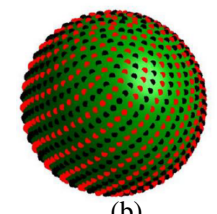

(b)
Fig. 4. Direction sampling schemes for a near transmitter. (a) Cube based sampling. (b) Spiral sampling.

3) Diffraction: Launching from a diffraction edge is also implemented with OptiX 2-D launching. In this case, one dimension corresponds to the launch points on the edge and another dimension corresponds to the angle $\alpha$, which controls the sampling of the Keller cone. Let $\hat{\mathbf{t}}$ denote the direction vector of the edge. Directions of the diffracted rays, $\mathbf{s}_{\mathrm{d}}$, at certain edge point can be computed using

$$
\mathbf{s}_{\mathrm{d}}(\alpha)=-\cos (\beta) \hat{\mathbf{t}}-\sin (\beta)\left(\cos (\alpha) \mathbf{i}_{\mathrm{t}}+\sin (\alpha) \mathbf{i}_{\mathrm{n}}\right)
$$

where $\beta=\arccos \left(-\mathbf{s}_{\mathrm{i}} \cdot \hat{\mathbf{t}}\right), \mathbf{i}_{\mathrm{n}}=\mathbf{s}_{\mathrm{i}} \times \hat{\mathbf{t}}$ and $\mathbf{i}_{\mathrm{t}}=\mathbf{i}_{\mathrm{n}} \times \hat{\mathbf{t}}$. Sampling is illustrated in Fig. 5a. The relevant sampling angles $\alpha$, i.e., those corresponding to non-penetrating directions are detected by analysis of the geometry of adjacent surfaces. Fig. 5b illustrates the sampling for multiple edge points, when the incident rays originate from a single point.

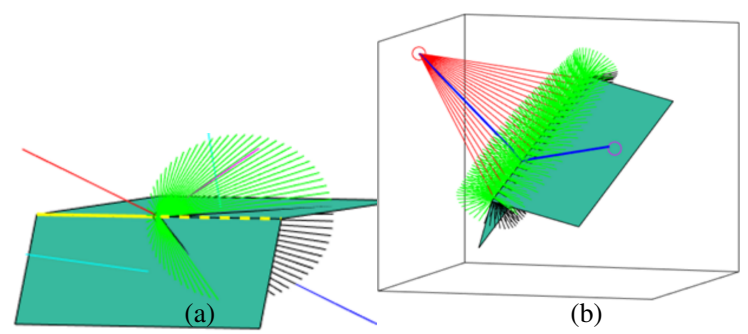

Fig. 5. Simulation of ray propagation at diffraction edges. (a) Keller cone sampling. Red line corresponds to the incident ray $\mathbf{s}_{\mathrm{i}}$ and green lines represent diffracting rays $\mathbf{s}_{\mathrm{d}}(\alpha)$; the blue line is the ray for $\alpha=0$. (b) 2-D ray launching from a diffraction edge using multiple edge points.

One subproblem is to adjust the resolution of the Keller cone sampling. In the case of a far transmitter, we relate it to the density of the planar wavefront sampling lattice. Let $\delta$ denote the distance between horizontally or vertically adjacent ray origins on the wavefront plane.
Then, if we consider line-of-sight (LOS) connections, the radius of the receiver sphere must be set to $\delta / \sqrt{2}$ so that the LOS ray can hit the sphere. However, in the case of Keller cone sampling, the rays diverge. If we want to guarantee that the receiver sphere is hit at a distance $d$ by one ray, we must use sufficiently dense sampling for angular directions, that is, spheres having radius $\delta / \sqrt{2}$ and placed on a circle with radius $d$ at regular intervals must overlap for the sampling resolution.

\section{E. Geometry}

In the current implementation, the geometry of the environment is approximated with a triangle mesh based representation. The mesh is enhanced by a wireframe, which gives the places of the potential diffraction edges. These edges are determined in a preprocessing step (Fig. 6).

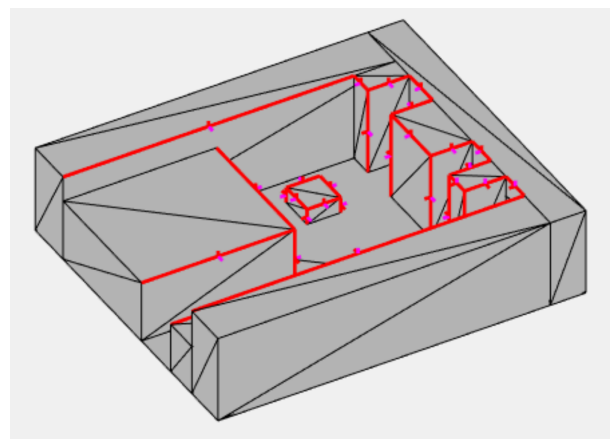

Fig. 6. An example of geometry representation, diffraction edges shown with red lines.

\section{GNSS Use Case}

A GNSS radio wave propagation simulation was done with the ray tracer in a scenario involving an urban area, which consisted of many businesses, recreations, narrow streets, and tall buildings close together (i.e., street canyons). In the scenario, twelve satellites were tracked. The satellite constellation and approximate 3$\mathrm{D}$ geometry of the situation along with the receiver trajectory is shown in Fig. 7. The satellite channel was the GPS L1 channel with the C/A pseudorandom code. The center frequency for this channel is $1.575 \mathrm{GHz}$ and its bandwidth is $24 \mathrm{MHz}$. The measurement device (LabSat 3 [21]) monitored a sub-band of $9.66 \mathrm{MHz}$. This sub-bandwidth is small compared to the center frequency and the differences in the electromagnetic calculations are marginal at different frequencies in this sub-band. Therefore only the center frequency of $1.575 \mathrm{GHz}$ was used in the electromagnetic analysis.

\section{A. Simulation Setup}

A rough 3-D model illustrated in Fig. 7 was developed to model a measurement scenario. The measurements were done in the area of Taka-Lyötty in the city of 
Oulu, Finland. The triangle mesh used to represent the buildings and the ground consisted of 2792 triangles and the number of the potential diffraction edges was 1520. The average location of the satellites over the measurement period was used as their fixed location (Fig. 7a). Ray path information was computed for 927 points of the approximate measurement route; every 8th sample point is shown in Fig. $7 \mathrm{~b}$.

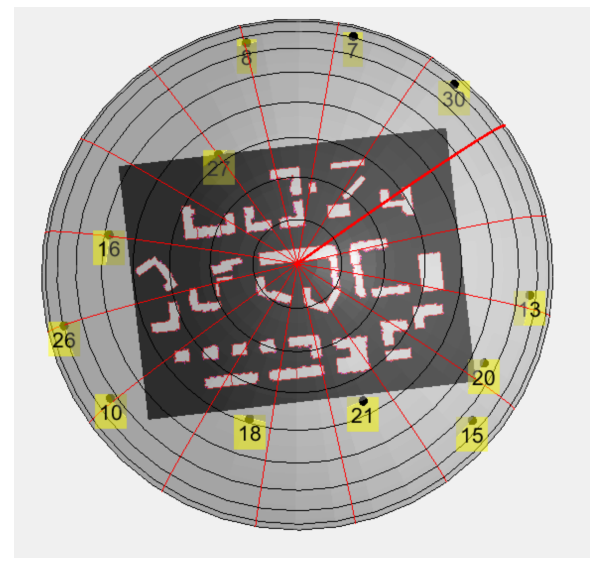

(a)

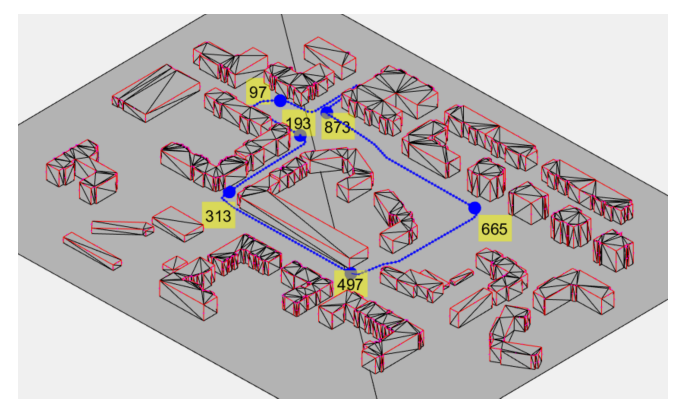

(b)

Fig. 7. Simulation scenario. (a) Positions of the satellites with respect to the origin of the building geometry. (b) Route of the receiver with labels of some sample point indices.

In the simulation, the maximum number of interactions on the ray path was set to 7 and one diffraction on the path was allowed. The size of the launching plane was set to $480 \times 480 \mathrm{~m}^{2}$ and the distance between adjacent sampling points was $20 \mathrm{~cm}$, which leads to $5.76 \times 10^{6}$ sample points. The path length parameter $d$, which partially controls the Keller cone sampling, was set to 250 meters. As a result, 4,632 ray directions were sampled for each cone (angular resolution $0.078^{\circ}$ ). To speed up simulation of diffractions, only the edges at the distance of 100.0 meters from the receiver location were used. For focusing, we used 3 iterations for the launching plane and Keller cones.

\section{B. Results}

A simulation example for the GNSS satellite PRN 15 is given in Fig. 8a. The notation PRN stands for the pseudo-random noise sequence or Gold code that each satellite uses to differentiate itself from other satellites. In absence of exact signal power information the amplitude of the field vector of a line of sight signal could chosen arbitrarily to be for example 1 . The result would be that the received signal power fluctuates more or less around $0 \mathrm{~dB}$ when there is a LOS signal present. Here the SNR axis has been shifted to such that the simulated and measured LOS signals more or less have the same values. The fluctuations depend on how the multipath components effect the LOS signal and also from what direction the signal is coming from. The patch antenna used for measurements and its model used for simulations and was oriented so that it not have much gain at low elevation angles. The simulated power plotted in Fig. $8 \mathrm{a}$ corresponds to Fig. $8 \mathrm{~b}$ plotting the measured SNR profile. The deep fades in the signal correspond mainly to diffractions without reflections present. According to theory, the diffracted signal powers are much less than what the measurements indicate. The compressed measurement range is caused by the receiver. Also there are fluctuations in the measurements not re-produced in the simulations. These mostly seemed to be arising from shadowing and scattering caused by trees and other smaller details not present in the simulation map. After adding a fixed scaling term in $\mathrm{dB}$ to the simulated profile it matched the measurement SNR profile well.

The proportion of the field power lost in diffuse scattering is taken into account by multiplying the reflection and diffraction coefficients by a constant $\leq 1$. Here the power loss is modelled by multiplying reflection and diffraction coefficients by the coefficient $\sqrt{\beta}$, where $\beta$ is the power proportion of the signal going into the reflection or diffraction. According to [22], $\beta=0.5$ is appropriate for office environments and $\beta=0.7$ fits rural environment measurements. We have chosen $\beta=0.5$ conforming with [23]. A generic relative material permittivity of $\varepsilon=4.5$ has been chosen for the simulation.

The performance of path detection is illustrated in Fig. 9, which shows the numbers of paths found for each satellite and route sample point. To highlight our observation that the characteristics of the path collection depend on the satellite altitude, we group the plots of the satellites into three sets. It can be seen that the number of paths tends to be lower for the satellites at higher altitudes. Numerically, for the satellites with the highest altitude, $\operatorname{PRN}\{16,18,21,27\}$, the median path count is in the range $12-18$, for $\operatorname{PRN}\{8,10,15,20\}$ it is in the range 18-32, and for PRN $\{7,13,26,30\}$ (lowest altitude) in the range 32-57. The reason for this may be that in the case of high altitude, a ray bounces more easily back to the sky, whereas in the case of low altitude it can go through several reflections. This notion is also supported by the fact, that the numbers of paths have peaks at the receiver points in the range 265-280. At that time the receiver is located in a street canyon (recall Fig. 7b), which supports 


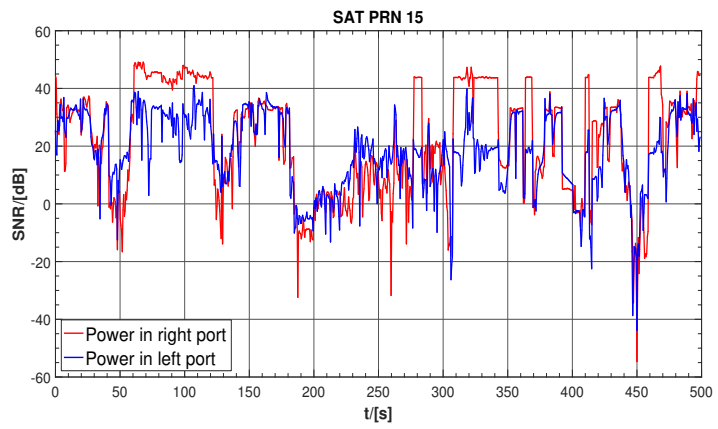

(a)

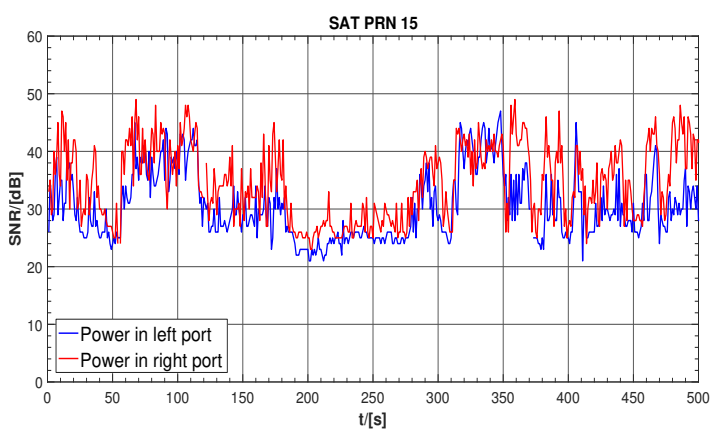

(b)

Fig. 8. SNR profile simulation for GPSS satellite PRN 15. a) Simulated power in antenna ports.

b) SNR profile measurements done in Taka-Lyötty, Oulu.

bouncing of the ray between side walls.

Complementary information on ray tracing performance is shown in Fig. 10, which shows which type of dominating connection has been found in simulations between the transmitter and receiver. Considering the satellites in the highest altitude range, the LOS connection is established for PRN16 and PRN27, respectively, in $89.9 \%$ and $97.4 \%$ of cases, whereas for PRN18 and PRN21 it is obtained only in $65.9 \%$ and $63.6 \%$ of cases due to shadowing by tall buildings. Then diffraction plays an important role in propagation.

\section{Conclusion}

This paper presents a ray tracing simulator and the characteristics of a GNSS channel in a specific urban area. The simulation results prove it to be an adequate tool for predicting the electromagnetic field in this instance. Also the use of GPU processing enables a considerable speedup of the path calculations, which is especially useful in modeling multiple interactions along a path. The number of paths found are accurate as they have been compared with results produced by other simulators. The simulation results indicate that the shadowing of buildings often cause diffractions to be the strongest signals for satellites low in the elevation angle in street canyon environments. Further computation speedup could be achieved by preprocessing the map database and is envisioned to be a continuation for this
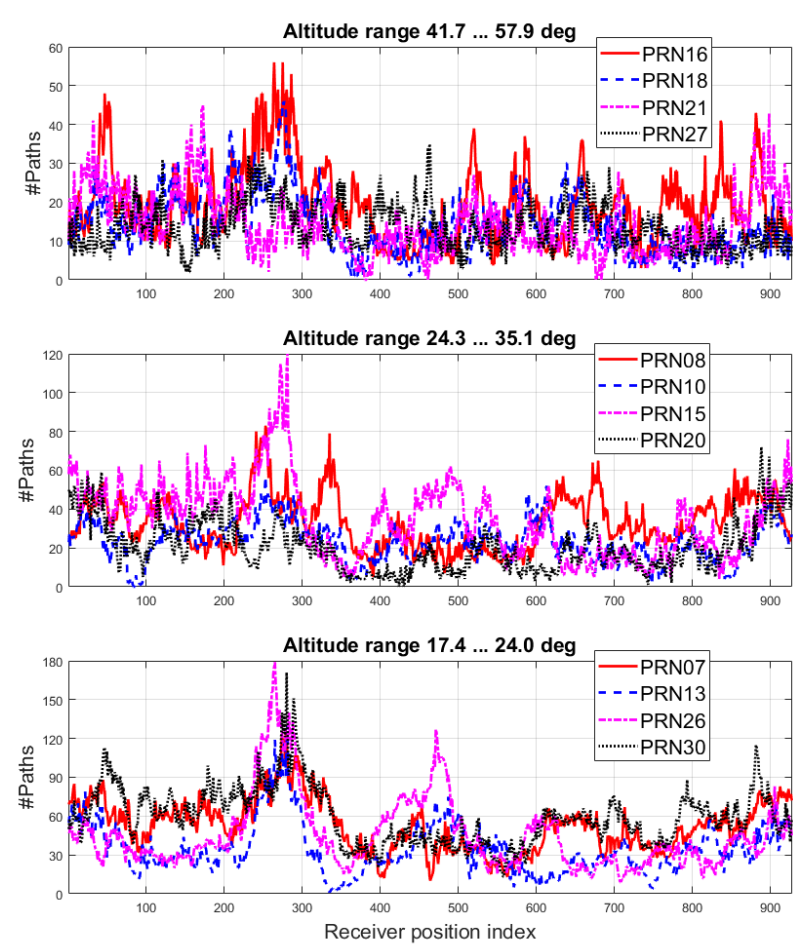

Fig. 9. The numbers of ray paths found for satellite/receiver point pairs. The satellites have been grouped to three sets according to separated ranges of altitudes. Those ranges are shown in the titles of the subplots.

research. In addition, EM calculations would be done faster once implemented by using CUDA. That would improve the execution speed of RT calculation since the analysis of signal power levels would allow early termination of path search.

\section{ACKNOWLEDGMENT}

This work was done under WERME and ENABLER projects, which have been funded by Business Finland. Preliminary work was done in Fullray Project funded by Nokia. In addition, this research has been financially supported in part by Academy of Finland 6Genesis Flagship (grant 318927). The authors would like to thank especially Jani Kallankari from Verkotan, Aki Hekkala and Seppo Salonen from Keysight and Jari Ylioinas and Eero Heikkinen from Nokia for support and collaboration.

\section{REFERENCES}

[1] M. Petovello, "What are the challenges of ray-tracing for GNSS applications?" InsideGNSS, pp. 32-37, November/December 2016.

[2] A. A. M. Saleh and R. Valenzuela, "A statistical model for indoor multipath propagation," IEEE Journal on Selected Areas in Communications, vol. 5, no. 2, pp. 128-137, February 1987.

[3] M. F. Iskander and Z. Yun, "Propagation prediction models for wireless communication systems," IEEE Transactions on Microwave Theory and Techniques, vol. 50, no. 3, pp. 662-673, Mar 2002. 

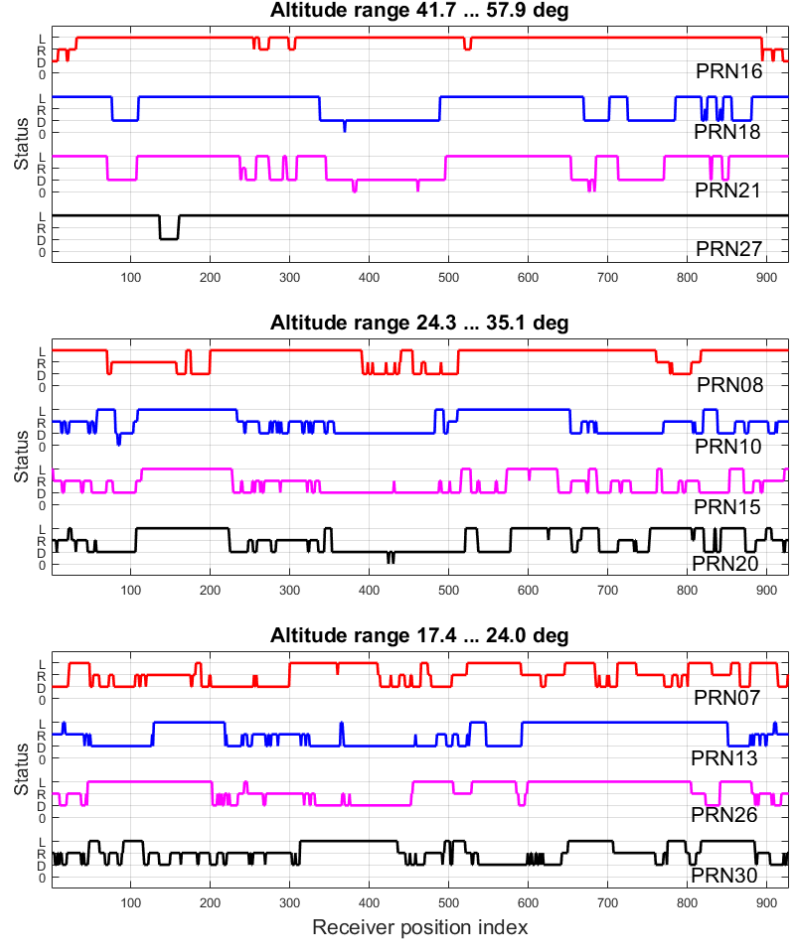

Fig. 10. Ray tracing status for satellite/receiver point pairs. L: LOS connection, R: Connection via reflections (no LOS), D: Connection just via paths containing diffraction, 0 : no paths found.

[4] R. G. Vaughan and J. B. Andersen, Channels, Propagation and Antennas for Mobile Communications, 2nd ed. The Institution of Engineering and Technology, 2017.

[5] S. G. Parker, J. Bigler, A. Dietrich, H. Friedrich, J. Hoberock, D. Luebke, D. McAllister, M. McGuire, K. Morley, A. Robison, and M. Stich, "OptiX: A general purpose ray tracing engine," in ACM SIGGRAPH Papers, 2010

[6] http://www.remcom.com/xf7-xstream/, accessed: 2016-11-01.

[7] C. Schissler, R. Mehra, and D. Manocha, "High-order diffraction and diffuse reflections for interactive sound propagation in large environments," ACM Transactions on Graphics, vol. 33, 2014.

[8] R. Felbecker, L. Raschkowski, W. Keusgen, and M. Peter, "Electromagnetic wave propagation in the millimeter wave band using the NVIDIA OptiX GPU ray tracing engine," in Proc. European Conference on Antennas and Propagation (EUCAP), 2011, pp. 488-492.

[9] M. Schiller, A. Knoll, M. Mocker, and T. Eibert, "GPU accelerated ray launching for high-fidelity virtual test drives of VANET applications," 2015.

[10] P. Gegout, P. Oberlé, C. Desjardins, J. Moyard, and P. M. Brunet, "Ray-tracing of GNSS signal through the atmosphere powered by CUDA, HMPP and GPUs technologies," IEEE Journal of Selected Topics in Applied Earth Observations and Remote Sensing, vol. 7, no. 5, pp. 1592-1602, May 2014.

[11] C. A. Balanis, Advanced Engineering Electromagnetics. John Wiley and Sons, 2012.

[12] D. A. McNamara, C. W. I. Pistorius, and J. A. G. Malherbe, Introduction to the Uniform Geometrical Theory of Diffraction. Artech House Publishers, 1990.

[13] F. Yun and M. F. Iskander, "Ray tracing for radio propagation modeling: Principles and applications," IEEE Access, vol. 3, pp. 1089-1100, 2015.

[14] A. F. Molisch, H. Asplund, R. Heddergott, M. Steinbauer, and T. Zwick, "The COST259 directional channel model-part I: Overview and methodology," IEEE Transactions on Wireless Communications, vol. 5, no. 12, pp. 3421-3433, December 2006.
[15] J. B. Keller, "Diffraction by an aperture," Journal of Applied Physics, 1957.

[16] R. G. Kouyoumjian and P. H. Pathak, "A uniform geometrical theory of diffraction for an edge in a perfectly conducting surface," Proc. IEEE, pp. 1448-1461, 1974.

[17] P. H. Pathak and R. G. Kouyoumjian, "The dyadic diffraction coefficient for a perfectly conducting wedge," Ohio State University, 1970.

[18] V. Degli-Esposti, F. Fuschini, E. M. Vitucci, and G. Falciasecca, "Measurement and modelling of scattering from buildings," IEEE Transactions on Antennas and Propagation, vol. 55, no. 1, pp. 143-153, 2007.

[19] H. Ling, R. C. Chou, and S. W. Lee, "Shooting and bouncing rays: calculating the RCS of an arbitrarily shaped cavity," IEEE Transactions on Antennas and Propagation, vol. 37, no. 2, pp. 194-205, Feb 1989.

[20] G. Durgin, N. Patwari, and T. S. Rappaport, "An advanced 3D ray launching method for wireless propagation prediction," in 1997 IEEE 47th Vehicular Technology Conference. Technology in Motion, vol. 2, May 1997, pp. 785-789 vol.2.

[21] https://www.labsat.co.uk/index.php/en/, accessed: 2019-02-20.

[22] E. M. Vitucci, F. Mani, V. Degli-Esposti, and C. Oestges, "Polarimetric properties of diffuse scattering from building walls: Experimental parameterization of a ray-tracing model," IEEE Transactions on Antennas and Propagation, vol. 60, no. 6, pp. 2961-2969, June 2012.

[23] L. Raschkowski et al., "METIS channel models (D1.4)," https:/ www.metis2020.com/wp-content/uploads/METIS_D1.4_v3.pdf, accessed: 2018-10-09. 